\title{
The Effect of Neurofeedback Training on Increasing Sustained Attention in Veterans with Posttraumatic Stress Disorder
}

\author{
Neda Rastegar ${ }^{1}$, Behrouz Dolatshahi2 ${ }^{2 *}$, Ebrahim Rezaei Dogahe ${ }^{3}$ \\ 1. Department of Clinical Psychology, Islamic Azad University, Karaj Branch, Karaj, Iran. \\ 2. Department of Clinical Psychology, Substance Abuse and Dependence Research Center, University of Social Welfare and Rehabilitation Sciences, Tehran, Iran \\ 3. Department of Clinical Psychology, University of Social Welfare and Rehabilitation Sciences, Tehran, Iran.
}

ditation: Rastegar, N., Dolatshahi, B., \& Rezaei Dogahe, E. (2016). The effect of neurofeedback training on increasing sustained attention in veterans with posttraumatic stress disorder. Journal of Practice in Clinical Psychology, 4(2), 97-104. http://dx.doi.org/10.15412/J.JPCP.06040204

: http://dx.doi.org/10.15412/J.JPCP.06040204

\section{Article info:}

Received: 06 Dec. 2015

Accepted: 27 Feb. 2016

Keywords:

Neurofeedback, Sustained attention, Posttraumatic stress disorder, Veterans

\begin{abstract}
Objective: This study aimed to evaluate the effect of neurofeedback training on improving sustained attention of veterans with posttraumatic stress disorder (PTSD).

Methods: The research design is quasi-experimental with pretest-posttest and control group. The study population consisted of the veterans with PTSD who were hospitalized in psychiatric wards. Purposeful sampling method was done to select 30 patients in psychiatric hospitals of Sadr, Delaram, and Parsa during spring, summer, and autumn of 2014 by considering their arrivals and departures. Then, they were randomly assigned into 2 experimental $(n=15)$ and control group $(\mathrm{n}=15)$. The neurofeedback training by alpha-theta protocol was administered to the experimental group, but the control group did not receive any neurofeedback training. The sustained attention was measured by continuous performance test (CPT). After that, the data were analyzed by multivariate analysis of variance.
\end{abstract}

Results: The results showed that neurofeedback training significantly increased the omission errors $(\mathrm{P}<0.001, \mathrm{~F}=17.074)$, commission errors $(\mathrm{P}<0.001, \mathrm{~F}=18.515)$, and reaction times $(\mathrm{P}=0.044, \mathrm{~F}=4.511)$ in sustained attention and reduced correct detection.

Conclusion: According to the findings, the relation between alpha and theta waves, and based on underlying principles, neurofeedback treatment has achieved acceptable results.

\section{Introduction}

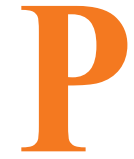

osttraumatic stress disorder (PTSD) is an anxiety disorder with $8 \%$ prevalence in all aspects of an American person's life (Kessler, Sonnega, Bromet, Hughes, \& Nelson, 2002). This disorder consists of reactions a person divulges in the face of uncontrolled stresses like war, natural disasters, physical abuse, and so on (Kulka et al., 1990; Yehuda, 2002). For a long time, the incidence of psychological distresses was discussed and studied after experiencing a traumatic event (Nawijn et al., 2015). People such as militaries who were exposed to more traumas show higher incidence of PTSD (Norris, 1990; Kang, Natelson,

* Corresponding Author:

Behrouz Dolatshahi, PhD

Address: Department of Clinical Psychology, Substance Abuse and Dependence Research Center, University of Social Welfare and Rehabilitation

Sciences, Tehran, Iran.

Tel: +98 (912) 2890655

E-mail:dolatshahee@yahoo.com 
Mahan, Lee, \& Murphy, 2003). Generally, war is the most frequent cause of PTSD (Tanielian \& Jaycox, 2008).

Experiencing war and intense pressure causes emotional disorders and since the recognition and excitement are associated with each other, cognitive disorders (loss of memory and attention biases) will appear after emotional disorders (Buckley, Blanchard, \& Neill, 2000).

Patients with PTSD often complain of difficulty in concentration, attention, and memory. Poor performance on tests of attention, memory, and other cognitive skills were attributed to PTSD in many studies (Crowell, Kieffer, Siders, \& Vanderploeg, 2002). Probably, one of the crooked infrastructures of this disorder that may lead to other symptoms is cognitive disorder (memory and attention) (Wild \& Gur, 2008). Low performance on neurocognitive tasks may have particular relevance with PTSD, but several studies have proven the reduction of hippocampus volume (Gilbertson et al., 2002; Villarreal et al., 2002).

In McNally's study (1997), cognitive abilities that are more than average have been proposed as a protective factor against PTSD and on the other hand, less than average cognitive abilities have been proposed as predisposing factors for PTSD in individuals exposed to trauma (McNally, 1997). Neurological studies support this theory that stress causes damage to the hippocampus (Bremner, 2001). Also, there are flaws in the activity of these brain regions explaining difficulties of memory and concentration in patients with PTSD (Weber et al., 2005).

Therefore, according to the proposed record, one of the main problems that people with PTSD are involved in is difficulty in attention. These deficiencies play an important role in establishing and continuation of the PTSD symptoms. So the methods and techniques that can improve attention are very important.

In this regard, various nonpharmacological methods such as eye movement desensitization and reprocessing, cognitive behavioral therapy, long-term exposure therapy, cognitive trauma-focused cognitive behavioral therapy, and cognitive restructuring have been tried. Among these, neurofeedback training is a new technique that has been recently used to improve memory. Neurofeedback is used to teach individuals make normal the reaction of brain waves (Mann, Lubar, Zimmerman, Miller, \& Muenchen, 1992).

Many studies have shown that neurofeedback is effective on changing the brain function and thus induces important improvements in clinical symptoms in many disorders such as epilepsy, attention deficit disorder, hyperactivity, learning disorders, and mental injuries (Hammond, 2005). The effectiveness of nneurofeedback training has been reported in many other studies for various situations like alcohol abuse (Pattinson \& Kulkosky, 1998), PTSD (Peniston \& Kulkosky, 1991), anxiety disorders (Moore, 2000), depression (Hammond, 2003), trait anxiety (Hardt \& Kamiya, 1978), Attention deficit hyperactivity disorder (Othmer, 2001), as well as improving the performance of athletes and increasing their physical balance (e.g. gymnastics and skiing) (Hammond, 2007). Also, the research results showed that neurofeedback improves attention process (Egner \& Gruzelier, 2004), accuracy in working memory test (Vernon et al., 2003), and the performance on mental rotation test (Hanslmayr, Sauseng, Doppelmayr, Schabus, \& Klimesch, 2005).

Overall, as Masterpasqua (2000) concluded, neurofeedback may be used in some psychiatric conditions as a substitute for drug therapy. But, generally it should be considered as a component of a multi-dimensional approach. When the neurofeedback is being used with medication, it can improve clinical outcomes in some patients (Masterpasqua \& Healy, 2003).

However, the studies in this field were mostly based on single-case studies and we need more research in this subject. Regarding the studies on the effectiveness of neurofeedback training on memory and posttraumatic stress disorder, the current study seeks to evaluate the effect of neurofeedback training on improving sustained attention on veterans with PTSD.

\section{Methods}

The design study is quasi-experimental with pretestposttest and control group. In this study, sustained attention was considered as the dependent variable and treatment (neurofeedback) has been considered as an independent variable.

The study population consisted of all veterans with PTSD hospitalized in psychiatric hospitals. Sampling was done by purposeful method among the patients that had been hospitalized in psychiatric centers of Sadr, Delaram, Parsa during spring, summer, and autumn of 2014. Cohen's method was used to determine the sample size. In this method, $\alpha$ was determined as 0.05 and effect volume as 0.50 . In this case, with subjects potency of 0.76 people can be selected on each groups. To select the sample members, all psychiatric cases were checked and through preliminary assessment based on diagnostic interview and obtained scores in BDI-II and PCL questionnaires and inclusion criteria, 30 male volunteered (aged 40 to 
$60)$ were selected. Then, they were randomly divided into intervention group $(n=15)$ and control group $(n=15)$.

Inclusion criteria for selecting sample were: definite diagnosis of posttraumatic stress disorder after PCL test and clinical psychiatrist's interview based on DSM-IVTR criteria, being male, being 40 to 60 years, receiving a fixed dose of drug, being literate.

Exclusion criteria were as follows: having a diagnosis of psychosis or substance abuse, obtaining a score higher than 29 on the BDI-II test, having already been treated with neurofeedback, getting same treatment to improve attention at the same time, having severe physical diseases that intervene in the process of research, and being absent more than 3 days during the intervention.

The Posttraumatic Stress Disorder Checklist (PCL): PTSD list is a self-report scale applied for evaluating and screening the patients. Although the list has been only validated on armed forces, it is concise and practical. It was prepared by Verz, Herman, Huesca and Kane (1994) based on DSM-IV criteria that covers 17 items, including 5 items about re-experiencing symptoms of PTSD, 7 items related to the symptoms of numbness and avoidance, and 5 items related to symptoms of arousal.

This list has 3 editions as follows: PCL-M edit military in conjunction with traumatic war stress, normal or ordinary edit of PCL-C in relation to non-combat stress or sor's normal (the public), and special edit of PCL-S in relation to problematic events related to stressful situations.

The validity of this list in Iran was calculated by Goudarzi (2003) at Shiraz University by using the Cronbach $\alpha$ coefficient of obtained data from implementation of this list on 117 subjects. This ratio was $93 \%$ and showed the list validity. Also, the reliability coefficient of the list is equal to $87 \%$ obtained through composition method (based on odd or even items) and shows the list validity. In order to provide index for the validity of this scale, its correlation was calculated with the list of life events. The correlation coefficient was $\mathrm{r}=0.37$ $(\mathrm{n}=117, \mathrm{P}=0.0001)$ that indicates concurrent validity. In PCL questionnaire, questions 1-5 evaluate the re-experiencing scale, 6-12 evaluate the numbing scale and questions, and 13-17 evaluate the scale of more excitation. Each question has been scored from 1 to 5 .

Mirzaee, Imani (2011) implemented PTSD list on 475 psychiatric veterans to find its validity. In this regard, 50 of them were re-evaluated during 2 weeks (Mirzaee \& Imani, 2011). The Pearson correlation coefficient was
0.866 that showed the stability by retest method. The Cronbach $\alpha$ obtained for the 2 PTSD-I and PCL-M tests that simultaneously was done on 20 patients was 0.866 .

Continuous Performance Test (CPT): This test was the most common way of measuring inhibition and attention in 1990s. This test was initially used to measure brain injury but gradually its application was expanded and used for other purposes. One of the primary applications of this test was measuring the attention deficits in patients with schizophrenia and the effects of drug treatments on the process of attention on these patients. The purpose of this test is to determine the sustained attention in children. The main approach is to randomly show target motive on the screen among other motives.

The subjects are taught to press a button whenever target appears. Its variables include: 1) Commission errors: the number of error in assignment that is arousal index (subjects' responses to non-target motives considered as an assignment error); 2) Omission errors: the number of omitted that is attention index (when the subject loses its purpose, omission error occurs); and 3) Reaction time: the time of subject's firing between the purpose and response. Thirty years history of test indicates that it is a good tool for measuring the care, attention, and meditation. In this study, this test was used to evaluate "sustained attention".

The validity of this test has been approved by the criterion validity and its reliability has been reported between 0.52 and 0.93 . Different parts of the test had been implemented by Najarian, Shekarshekan, Mehrabizadeh on 43 elementary school boys during 20 days. The range was between 0.59 and 0.93 . All sign were calculated in 0.001 , if correlation coefficients were not obtained. The validity of the test was obtained by means of criterion validity and also by comparing the normal group (30 boys students' school) and hyperactive with Attention deficit (25 boys students' school). Statistical comparison of the two groups indifferent parts of the test showed the significant difference between two groups $(\mathrm{P}<0.001)$.

Beck Depression Inventory-II (BDI -II): This questionnaire is the revised form of Beck depression questionnaire developed to measure the severity of depression. This questionnaire has been adapted by depression criteria in $4^{\text {th }}$ edition of statistical recognition guide of Mental Disorders DSM-IV-TR. The test consists of 21 items related to various landmarks and its responses range between 0 and 30 . The questionnaire has been psychometrically re-evaluated several times since its introduction. The result of a meta-analysis revealed that the internal consistency of the tool varied between 0.73 
and 0.86 . The internal consistency of the instrument for the Iranian students is 0.87 while its reliability is 0.73 through test-retest procedure. The questioner validity and its construct validity was examined and confirmed through factor analysis procedure through employing a sample of 353 subjects by Dobson and Mohammadkhani, 2006 (Dobson \& Mohammadkhani, 2006).

At first, necessary permits re obtained wfrom authorities of Parsa, Delaram, and Sadr hospitals. Then, according to medical records, the definitive diagnosis of posttraumatic stress disorder based on diagnostic criteria DSM-IV-TR, clinical psychologist and psychiatrist interview, as well as PCL and BDI-II questionnaires (the subjects obtained score of upper than 50 in PCL questionnaire and lower than 29 in BDI-II questionnaire), a total of 30 male veterans aged 40 to 60 were selected (17 people from Delaram center, 10 people from Parsa hospital, and 3 people from Sadr hospital). Then, 15 patients were randomly assigned to the experimental group and 15 patients to the control group. Both groups were tested before the intervention (CPT test). Neurofeedback training was given to the experimental group in 20 sessions for 45 minutes by alpha-theta protocol on PZ area and the control group were just under normal treatment.

The method of neurofeedback was as follow: At first, electrodes were set on desired location with special gelsin 10-20 standard points according to international system.

Auditory feedbacks are in alpha/theta protocol, including river and ocean sounds. River sound was a feedback from alpha wave prepotency while ocean sound was a feedback from theta wave prepotency. All of them were optimal. The subjects should hear ocean sound more than others and think about bad memories and ideals in their lives and the solutions of their problems. In this task, there was no reinforcement or suppression, but subjects needed to justify (mask harmony) between alpha/theta waves. The subjects should think about the solutions of their bad experiences. However, delta axis alerted by wave controlling when patients approach the state of drowsiness, as they must be conscious during the process. At the end, posttest was taken. In order to analyze the data, multivariate analysis of variance (MANOVA) was used.

\section{Results}

After collecting the data, SPSS version 22, was used to analyze them. Regarding descriptive statistics, the mean and standard deviation were used. To check the consistency of the 2 groups regarding their age, marital status, and education, the independent $t$ test and statistical test $\chi^{2}$ were used. To check the questions in the survey research, MANOVA was used.

The average age of participants was 48.20 years and ranged from 43 to 60 years. Although, the mean age in the control group was higher (48.73 years) and ranged from 41 to 60 years, this difference was not statistically significant $(\mathrm{P}=0.739, \mathrm{t}=0.336)$. Also, there was no significant difference between groups regarding their marital status ( $\left.\mathrm{P}=0.699, \chi^{2}=715\right)$. This lack of difference between the two groups was also visible in terms of their education level $\left(\mathrm{P}=0.649, \chi^{2}=1.644\right)$.

Table 1. For frequency and cumulative frequency of Education, Age, and marital status for different group of tests.

\begin{tabular}{|c|c|c|c|c|c|}
\hline \multicolumn{6}{|c|}{ Experimental group } \\
\hline Education & Frequency & Cumulative frequency & Percent of cumulative frequency & Average age & *Material status \\
\hline Primary school & 3 & 20.0 & 20.0 & 51.67 & $M(3)$ \\
\hline Middle school & 4 & 26.7 & 46.7 & 44.5 & $S(2), M(2)$ \\
\hline High school & 1 & 6.7 & 53.3 & 43 & $M(1)$ \\
\hline Diploma & 7 & 46.7 & 100.0 & 49.57 & $M(4), D(3)$ \\
\hline Total & 15 & 100.0 & & & \\
\hline \multicolumn{6}{|c|}{ Control group } \\
\hline Education & Frequency & Cumulative frequency & Percent of cumulative frequency & Average age & *Material status \\
\hline Primary school & 2 & 13.3 & 13.3 & 49.5 & $M-D$ \\
\hline Middle school & 5 & 33.3 & 46.7 & 50.6 & $S(1), M(3), D(1)$ \\
\hline High school & 3 & 20.0 & 66.7 & 46.67 & $M(3)$ \\
\hline Diploma & 5 & 33.3 & 100.0 & 47.8 & $M(5)$ \\
\hline Total & 15 & 100.0 & & & \\
\hline
\end{tabular}


Table 2. The results of multivariate analysis of variance on posttest scores of sustained attention in experimental group and control group with pretest control.

\begin{tabular}{|cccccc}
\hline & Value & F & df Hypothesis & df Error & Significance level \\
\hline Pillai effect & 0.557 & 6.602 & 4 & 21 & 0.001 \\
\hline Wilks' Lambda & 0.443 & 6.602 & 4 & 21 & 0.001 \\
\hline Hetling effect & 1.258 & 6.602 & 4 & 21 & 0.001 \\
\hline The biggest root of error & 1.258 & 6.602 & 4 & 21 & 0.001 \\
\hline & & & & & PRACIICE in \\
\hline
\end{tabular}

Table 3. Analysis of sustained attention scores covariance in experimental and control groups after adjusting the pretest scores.

\begin{tabular}{cccccccc}
\hline & Dependent variable & Total squares & df & Mean of square & $\mathbf{F}$ & Significance level & Effect amount \\
\hline \multirow{4}{*}{ Group } & Commission errors & 51.64 & 1 & 51.64 & 18.51 & 0.000 & 0.435 \\
& Omission errors & 217.73 & 1 & 217.73 & 17.07 & 0.000 & 0.416 \\
& Correct detection & 2844.25 & 1 & 2844.25 & 4.54 & 0.043 & 0.159 \\
& Reaction time & 16687.73 & 1 & 16687.73 & 4.51 & 0.044 & 0.158 \\
\hline
\end{tabular}

In Table 1 as it can be seen, the mean values of commission errors, omission errors, and reaction time of the pretest and posttest have decreased. However, the number of correct detection has increased. In this hypothesis, as well as last hypothesis, analysis of covariance was used with respect to 2-level divided independent variable (the experimental group and the control group), the continuous dependent variable (sustained attention pretest scores), and change variable (sustained attention posttest scores). Mentioned analysis shows the difference between the average scores of sustained attention for the experimental group (neurofeedback) and control group after adjusting the first pretest scores. The results of variance analysis are presented in Table 2.

According to Table 2 results and tests significant levels, there is a significant difference between experimental and control groups at least in one of the dependent variables. To find out it, 4 analyses of covariance were conducted between 2 groups.

According to the results of Table 3, the values of omission errors, correct detection, commission errors and reaction time in experimental and control groups have significant differences. In other words, there are significant differences between adjusted averages of omission errors, correct detection, commission errors, and reaction time in posttest scores by eliminating the effect of pretest scores. Based on these findings, neurofeedback can reduce omission errors, commission errors, and reaction time in posttest while it increased correct detection value. The strongest effect of neurofeedback is on reducing commission errors. In fact,
$43 \%$ of the difference in experimental group and the control group on error response is due to the neurofeedback.

\section{Discussion}

This study aimed to determine the effectiveness of neurofeedback in increasing the sustained attention in veterans with PTSD. The results showed that neurofeedback training reduced the omission errors, commission errors, and reaction time, but it increased correct detection. These findings are similar to the findings of Duric, Assmus, Gundersen and Elgen (2012), Arns, Drinkenburg and Kenemans (2012), Doppelmayr and Weber (2011), Logemann, Lansbergen, Van Os, Böcker and Kenemans (2010), Gevensleben et al. (2010), Drechsler et al. (2007), Beauregard and Levesque (2006), Cho et al. (2004), Egner \& Gruzelier (2004), Vernon et al., (2003), and Damasio (1994). Overall, the evidence suggests that neurofeedback can have beneficial results in improving attention. However, most studies on neurofeedback trainings have focused on children with ADHD or attention deficit disorder (ADD) and increasing their attention. Duric et al., (2012) has shown the effectiveness of neurofeedback in children and adolescents with ADHD or ADD. Hillard (2012) showed that neurofeedback is an effective treatment for ADHD or ADD. Also, Arns, Drinkenburg and Kenemans (2012) showed that neurofeedback training significantly improves attention deficit, hyperactivity, impulsivity, and depression complaints. Doppelmayr and Weber (2011) also showed that neurofeedback increases the reaction rate and improving visual-spatial ability. 
In addition to these studies, the impacts of neurofeedback was shown on changes in the reaction time (Drechsler et al., 2007), hemispherical performance in left partial areas, and increase in attention and response time (Beauregard \& Levesque, 2006; Cho et al., 2004), main improvements in sustainable control of the symptoms of ADHD, lack of emotional instability, and amount of homework (Lubar, 2003).

To explain why neurofeedback therapy increases sustained attention in patients, we can point to the philosophy of protocol-based training alpha/theta. In fact, the review of the literature about alpha/theta protocol (based on increasing the ability to coordinate these 2 waves) shows that mentioned protocol increases individual's focus. According to these facts, neurofeedback training based on alpha/theta waves provides deep relaxation in the individual, like tranquility in meditation and other forms of mental alertness. Neurofeedback based on the alpha/theta reduces the arousal of the central nervous system and provides a unique conscious feeling in a person. Peniston and Kulkosky (1999) suggests that neurofeedback based on the alpha/theta waves is similar to EEG-based relaxation. In fact, treatment based on the alpha/theta protocol is similar to other relaxation therapies such as meditation (Peniston \& Kulkosky, 1999).

Studies show that people learn consciously to refuse alpha waves and have an effective role on its making. These people describe experiences that are similar to meditation, Zen, and yoga (Kamiya, 1968). Increasing alpha waves can enhance person's ability to perceive phenomena and increase the understanding and sustained attention by raising the level of consciousness (Wurker, 1996). In contrast, theta waves are associated with arousal of the central nervous system (Jacobs \& Friedman, 2004).

The kind of arousal (generated due to the theta waves) is similar to brain activities of the people who are meditating (Ivanovski \& Malhi, 2007). Ivanovski and Malhi (2007) reported that theta waves strongly liken to levels of meditative experience. Theta waves and their activity are associated with the response to unexpected stimuli (such as attention process and cognitive function). Research has shown that theta activity inhibits the activity of neurons interfering or irrelevant and accordingly increases person's sustained attention to process information (Harmony et al., 1996).

According to Albert (1999), theta waves are apparently involved in the process of attention by limiting of access to specific information during process in brain cortex. Based on the available evidence, theta waves are associated with higher cognitive processes that require sustained concentration and attention (Albert, 1999). Don
(1977) believed that theta and alpha waves are associated with the transfer of experiences in consciousness and its increase that can lead to insight, creativity, problem solving, and sustained attention.

Moreover, neurofeedback treatment in our study has been administered in PZ area that is important in terms of the impact on memory and attention. Evidence suggests that parietal areas, especially in central areas are involved in memory and attention control (Menon, 2011). According to the studies conducted in this area, the activity of executive functions is low in the parietal lobes of patients with PTSD (Falconer et al., 2008). Weber and colleagues (2005) argued that the parietal areas (including PZ) will play a key role in working memory and attention control and these areas are often impaired in patients with PTSD (Weber et al., 2005). So, neurofeedback training that has an important role in attention and concentration using the alpha/theta protocol in PZ area is a proper place for increasing sustained attention in patients with PTSD.

\section{Limitations of the study}

This study, despite its significant results, had several limitations and weaknesses that are mentioned below:

- Non-random selection of participants that limits the ability to generalize the study findings.

- Enrollment of just patients with PTSD that warrants its generalization to other patients.

- No final decision on the impact of long-term treatment, due to the discontinuation of the study at follow-up,

- Lack of placebo neurofeedback control, due to time constraints, and

- Use of qEEG-based protocol in this study, due to financial constraints and lack of access to the qEEG device.

\section{Study suggestions}

According to the results of this study, we suggest that both neurofeedback education and medical treatment for patients with PTSD be used in special medical centers.

As there are other therapies such as taking medicine and psychological treatments like mind-awareness accomplish (perform) on patients' memory and attention, this study suggests that the effectiveness of these treatment be compared to other medial approaches and consulting education.

Due to the positive effect of neurofeedback on increasing the attention and focus of patients who suffer from 
PTSD, this study proposes that the future studies consider other problems and symptoms of these patients as well.

Since the use of the follow up stage can lead to the better assessment, this study suggests that further research studies this stage. Moreover, others studies can study and investigate the impact of the number of medical sessions during the treatment time.

It is suggested that, in addition to concentrating on PZ area, the protocol of alpha and beta on other areas of brain be investigated and its result compared with the present results. Finally we suggest that the effect of placebo in others studies be controlled and its results be compared with those of the present study.

\section{Research ethics}

All participants of this study were assured about the confidentiality of their personal information. They also gave their written consent about the research. Also, participant were allowed to leave the study at any time. Moreover, the research method had no conflict with the Islamic, cultural, or legal principles.

\section{Acknowledgments}

We appreciate the sincere help of Parsa Hospital manager, Dr Mohammad Asgari, and medical personnel of Mental Center of Delaram, also Foundation of Martyrs and Veterans Affairs who helped us in the realization of this study and all the devotees participating in the study.

\section{References}

Albert, S. M., Michaels, K., Padilla, M., Pelton, G., Bell, K., Marder, K., \& et al. (1999). Functional significance of mild cognitive impairment in elderly patients without a dementia diagnosis. American Journal of Geriatric Psychiatry, 7(3), 213220.

Arns, M., Drinkenburg, W., \& Kenemans, J. L. (2012). The effects of QEEG-informed neurofeedback in ADHD: An open-label pilot study. Applied Psychophysiology and Biofeedback, 37(3), 171-180.

Cho, B. H., Kim, S., Shin, D. I., Lee, J. H., Min Lee, S., Young Kim, I., et al. (2004). Neurofeedback training with virtual reality for inattention and impulsiveness. CyberPsychology $\mathcal{E}$ Behavior, $7(5), 519-526$

Beauregard, M., \& Lévesque, J. (2006). Functional magnetic resonance imaging investigation of the effects of neurofeedback training on the neural bases of selective attention and response inhibition in children with attention-deficit/hyper- activity disorder. Applied Psychophysiology and Biofeedback, 31(1), 3-20.

Bremner, J. D. (2001). Hypotheses and controversies related to effects of stress on the hippocampus: An argument for stressinduced damage to the hippocampus in patients with posttraumatic stress disorder. Hippocampus, 11(2), 75-81.

Buckley, T. C., Blanchard, E. B., \& Neill, W. T. (2000). Information processing and PTSD: A review of the empirical literature. Clinical Psychology Review, 20(8), 1041-1065.

Crowell, T. A., Kieffer, K. M., Siders, C. A., \& Vanderploeg, R. D. (2002). Neuropsychological findings in combat-related posttraumatic stress disorder. Clinical Neuropsychologist, 16(3), 310-321.

Dobson, K., \& Mohammadkhani, P. (2006). [Psychoanalysis characteristic of Beck Depression inventory (Persian)]. Rehabilitation Journal, 29, 82-88.

Damasio, A. R. (1994). Descartes' error: Emotion, rationality and the human brain. New York: Putnam.

Don, N. S. (1977). The transformation of conscious experience and its EEG correlates. Journal of Altered States of Consciousness, $3(2), 1077-1078$

Doppelmayr, M., \& Weber, E. (2011). Effects of SMR and theta/ beta neurofeedback on reaction times, spatial abilities, and creativity. Journal of Neurotherapy, 15(2), 115-129.

Drechsler, R., Straub, M., Doehnert, M., Heinrich, H., Steinhausen, H. C., Brandeis, D. (2007). Controlled evaluation of a neurofeedback training of slow cortical potentials in children with attention deficit/hyperactivity disorder (ADHD). Behavioral and Brain Functions, 3(1), 30-37.

Duric, N. S., Assmus, J., Gundersen, D., \& Elgen, I. B. (2012) ADHD children and adolescent-neurofeedback treatment: A randomized controlled clinical trial-effect of core symptoms. Paper presented at International conference and exhibition on Neurology \& Therapeutics, Embassy Suites Las Vegas, USA, 14-16 May 2012.

Egner, T., \& Gruzelier, J. H. (2004). EEG biofeedback of low beta band components: frequency-specific effects on variables of attention and event-related brain potentials. Clinical Neurophysiology, 115(1), 131-139.

Falconer, E., Bryant, R., Felmingham, K. L., Kemp, A. H., Gordon, E., Peduto, A., et al. (2008). The neural networks of inhibitory control in posttraumatic stress disorder. Journal of Psychiatry and Neuroscience, 33(5), 413-422.

Gevensleben, H., Holl, B., Albrecht, B., Schlamp, D., Kratz, O., Studer, P., et al. (2010). Neurofeedback training in children with ADHD: 6-month follow-up of a randomized controlled trial. European Child \& Adolescent Psychiatry, 19(9), 715-724.

Gilbertson, M. W., Shenton, M. E., Ciszewski, A., Kasai, K., Lasko, N. B., Orr, S. P., et al. (2002). Smaller hippocampa volume predicts pathologic vulnerability to psychological trauma. Nature Neuroscience, 5(11), 1242-1247.

Goudarzi, M. (2003). Validity and reliability of post-traumatic stress Mississippi scale (scale). Psychological Review, 26(2), 2528.

Hammond, D. C. (2003). An introduction to neurofeedback. Salt Lake City: University of Utah. 
Hammond, D. C. (2005). Neurofeedback with anxiety and affective disorders. Salt Lake City: University of Utah.

Hammond, D. C. (2007). Neurofeedback for the enhancement of athletic performance and physical balance. Journal of the American Board of Sport Psychology, 1(1), 1-9.

Hanslmayr, S., Sauseng, P., Doppelmayr, M., Schabus, M., \& Klimesch, W. (2005). Increasing individual upper alpha power by neurofeedback improves cognitive performance in human subjects. Applied Psychophysiology and Biofeedback, 30(1), 1-10.

Hardt, J. V., \& Kamiya, J. (1978). Anxiety change through electroencephalographic alpha feedback seen only in high anxiety subjects. Science, 201(4350), 79-81.

Harmony, T., Fernández, T., Silva, J., Bernal, J., Díaz-Comas, L. Reyes, A., et al. (1996). EEG delta activity: An indicator of attention to internal processing during performance of mental tasks. International Journal of Psychophysiology, 24(1), 161-171.

Hillard, B. (2012). Analysis of EEG rhythms using custom-made MatLab application for processing of data collected during neurofeedback training in ADHD subjects (BS thesis). Kentucky: University of Louisville.

Ivanovski, B., \& Malhi, G. S. (2007). The psychological and neurophysiological concomitants of mindfulness forms of meditation. Acta Neuropsychiatrica, 19(2), 76-91.

Jacobs, G. D., \& Friedman, R. (2004). EEG spectral analysis of relaxation techniques. Applied Psychophysiology and Biofeedback, 29(4), 245-254.

Kamiya, J. (1968). Conscious control of brain waves. Psychology Today, 1, 57-60.

Kang, H. K., Natelson, B. H., Mahan, C. M., Lee, K. Y., \& Murphy, F. M. (2003). Post-traumatic stress disorder and chronic fatigue syndrome-like illness among Gulf War veterans: A population-based survey of 30,000 veterans. American Journal of Epidemiology, 157(2), 141-158.

Kessler, R. C., Sonnega, A., Bromet, E., Hughes, M., \& Nelson, C. B. (2002). Posttraumatic stress disorder in the national comorbidity survey. Archives of General Psychiatry, 12(2), 1048-60.

Kulka, R. A., Schlenger, W. E., Fairbank, J. A., Hough, R. L., Jordan B. K., Marmar, C. R., et al. (1990). Trauma and the Vietnam War Generation: Report of Findings from the National Vietnam Veterans Readjustment Study. New York: Brunner/Mazel.

Logemann, H. N., Lansbergen, M. M., Van Os, T. W., Böcker, K. B., \& Kenemans, J. L. (2010). The effectiveness of EEG-feedback on attention, impulsivity and EEG: A sham feedback controlled study. Neuroscience Letters, 479(1), 49-53.

Lubar, J. F. (2003) Neurofeedback for the management of attention deficit disorders. In M. S. Schwartz \& F. Andrasik (Eds.), Biofeedback: A practitioner's guide ( $3^{\text {rd }}$ ed., pp. 409-437). New York: Guilford Press.

Mann, C., Lubar, J., Zimmerman, A., Miller, C., \& Muenchen, R. (1992). Quantitative analysis of EEG in boys with Attention deficit/hyperactivity Disorder: A Controlled Study with Clinical Implication. Pediatric Neurology, 8, 30-36.

Masterpasqua, F., Healy, K. N. (2003). Neurofeedback in psychological Practices. Professional Psychology: Research and Practice, 34(6), 652-656.
McNally, R. J. (1997). Implicit and explicit memory for traumarelated information in PTSD. Annals of New York Academy of Sciences, 821(1), 219-224.

Menon, V. (2011). Large-scale brain networks and psychopathology: A unifying triple network model. Trends in Cognitive Sciences, 15(10), 483-506.

Mirzaee, J., \& Amini, S. (2011). Validation list on the physical and psychological effects of war-related PTSD veterans. Paper presented at the $3^{\text {rd }}$ Congress of Clinical Psychology, Tehran, Imam Khomeini Hospital, Iran.

Moore, N. C. (2000). A review of EEG biofeedback treatment of anxiety disorder. Clinical Electroencephalograph, 31(1),1-6.

Nawijn, L., Van Zuiden, M., Frijling, J, L, Koch, S. B., Veltman, D. J., \& Olff, M. (2015). Reward functioning in PTSD: A systematic review exploring the mechanisms underlying anhedonia. Neuroscience \& Bio behavioral Reviews, 51, 189-204.

Norris, F. H. (1990). Screening for traumatic stress: A scale for use in the general population. Journal of Applied Social Psychology, 20, 1704-18.

Othmer, S. (2001). The promise of neurofeedback. The Brian Othmer Foundation. Retrieved from http://www.brianothmerfoundation.org/promiseofneurofeedback.htm.

Peniston, E. G., \& Kulkosky, P. J. (1999). Neurofeedback in the treatment of addictive disorders. In J. R. Evans \& A. Arbarbanel (Eds.), Introduction to quantitative EEG and Neurofeedback (pp. 157-179). San Diego, CA: Academic Press.

Peniston, E. G., \& Kulkosky, P. J. (1991). Alpha-theta brainwave neuro-feedback therapy for Vietnam veterans with combatrelated post-traumatic stress disorder. Medical Psychotherapy, $4(1), 47-60$

Tanielian, T., \& Jaycox, L. H. (2008). Invisible wounds of war: Psychological and cognitive injuries: Their consequences, and services to assist recovery. Santa Monica, CA: Research and Development.

Vernon, D., Egner, T., Cooper, N., Compton, T., Neilands, C., Sheri, A., et al. (2003). The effect of training distinct neurofeedback protocols on aspects of Cognitive Performance. In ternational Journal of Psychophysiology, 47(1), 75-85.

Villarreal, G., Hamilton, D. A., Petropoulos, H., Driscoll, I., Rowland, L. M., \& Griego, J. A. (2002). Reduced hippocampal volume and total white matter volume in posttraumatic stress disorder. Biological Psychiatry, 52(2), 119-25.

Weber, D. L., Clark, C. R., McFarlane, A. C., Moores, K. A., Morris, P., \& Egan, G. F. (2005). Abnormal frontal and parietal activity during working memory updating in post-traumatic disorder. Psychiatry Research, 140(1), 27-44.

Wild, J., \& Gur, R. C. (2008). Verbal memory and treatment response in post-traumatic stress disorder. Psychiatry, 193(3) 254-265.

Wurker, M. (1996). Awakening and EEG biofeedback. Biofeedback, 3, 18-22.

Yehuda, R. (2002) Post-traumatic stress disorder. New England Journal of Medicine, 346(2), 108-14. 